\title{
Physiological \\ Comorbidity Care: \\ The Unmet Need in \\ Patients with Chronic \\ Lung Diseases
}

\section{Lenos Archer-Diaby}

Editorial Assistant

Citation: EMJ Respir. 2020;DOI/10.33590/emjrespir/201102

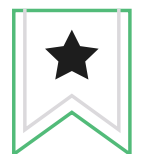

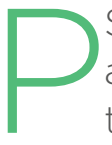
SYCHOLOGICAL distress significantly impacts patient quality of life (QoL), treatment adherence, mortality, and morbidity. However, patient distress is often under supported throughout a patient's treatment journey. The virtual European Respiratory Society (ERS) International Congress 2020 session 'Fear and Anxiety, Psychological Wellbeing and Prevention of Psychological Distress in Chronic Lung diseases,' underscored the importance of addressing these needs in chronic obstructive pulmonary disease (COPD) and idiopathic pulmonary fibrosis (IPF) patients, focussing on disease-specific fears, cognitive therapies, palliative care, and the importance of empathy.
\end{abstract}

\section{UNMET PSYCHOLOGICAL NEEDS: A PATIENT PERSPECTIVE}

"At diagnosis we felt robbed. We felt robbed hearing that news of 3-5 years," Ron Flewett stated, recalling the memory of his IPF diagnosis at the age of 53 years when he was informed of his short life expectancy. Flewett, who is now 59 years old, introduced the session in a patientperspective video that echoed throughout the entirety of the session. He continued: "Those days were quite dark for us and our anxiety levels were extremely high. I just felt so alone and so desperate at that point having no one to turn to for help." These psychological feelings heavily burdened Flewett and led him to contact his local hospice where he was offered Thai Chi and relaxation classes, and met other patients with other diseases with whom he could share his experiences with. However, apart from the hospice, support was not attainable until he deteriorated and lost $10 \%$ of his lung capacity. Upon visiting his interstitial lung disease nurse, the nurse questioned the mental wellbeing of Flewett and his wife, prompting both to burst into tears, as this was the first time that someone had genuinely cared to listen.

After several years of coping with the stresses and anxieties of his condition, Flewett emphasised that it is the healthcare professionals who need to encourage communication, as patients will not 


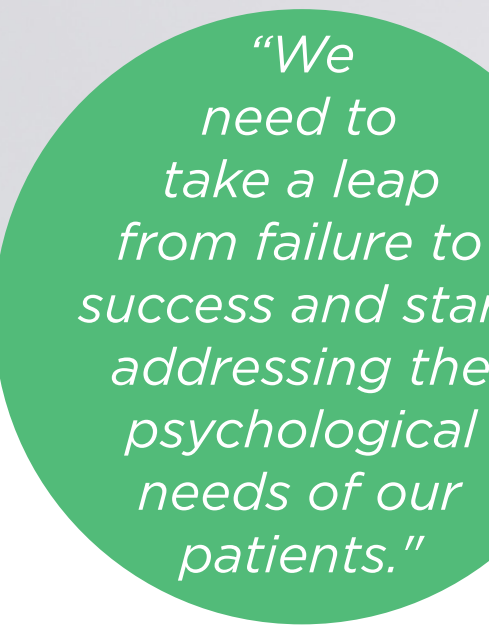
further advised to: it is okay to feel low and that there is no shame in seeking help. Put them in touch with other patients i.e., support groups or buddy systems and connect them with local charity organisations."

\section{THE IMPORTANCE OF MEASURING DISEASE-SPECIFIC FEAR}

Besides physiological comorbidities such as heart disease, diabetes, and osteoporosis, chronic lung diseases are often accompanied by psychological comorbidities including depression, loneliness, anxiety, and fear. Anxiety is one of the most common psychological comorbidities, reported among $10-55 \%$ of patients with COPD. Dr Thomas Reijnders, KU Leuven, Leuven, Belgium, a psychologist and physiotherapist specialising in behavioural changes in COPD rehabilitation, argued that measuring general anxiety fails to grasp the specificity that defines COPD as a disease and questioned whether measuring disease-specific fear is more relevant.

In a study that assessed the relationship between fear of physical activity and pulmonary rehabilitation (PR) outcomes in patients with a chronic lung disease, results demonstrated a correlation between increased fear of physical activity, shorter 6-minute walking distance, and decreased QoL. Conversely, lower levels of fear of physical activity correlated to improvements in 6-minute walking distance and QoL over the course of PR. Recent studies, including the one presented in this session, highlight that in COPD patients, disease-specific fears are related to dyspnoea perception. It is known that dyspnoea perception can lead COPD patients to avoid dyspnoea by reducing their physical activity levels, resulting in both physiological and psychological comorbidities.

In an experimental study investigating the impact of disease-specific fears on perception and neural processing of respiratory sensations, as well as the disease-specific fears and physical activity in daily life in COPD patients, results showed that increased attention to respiratory sensations is especially apparent in COPD patients with higher levels of dyspnoea-specific fear and is related to lower levels of physical activity in daily life. Concluding his talk, Dr Reijnders emphasised that: "Disease-specific fears should be taken into account when treating COPD patients to further improve the effects of PR."

\section{COGNITIVE THERAPY IN CHRONIC OBSTRUCTIVE PULMONARY DISEASE}

Presenting on a topic that healthcare professionals may not focus on but is certainly important for the patients, Dr Karen HeslopMarshall, Newcastle upon Tyne NHS Hospitals 
Foundation Trust, Newcastle, UK, discussed how addressing psychological wellbeing and how behavioural therapy delivered by respiratory nurses could help. Dr Heslop-Marshall reiterated how psychological comorbidities, such as anxiety and depression, are rarely assessed or treated, and that integration of physical and mental health is poor. "We have given patients treatment they don't need, and we have not given them treatment they do need," she claimed before describing the cognitive behavioural therapy (CBT) training model she developed specifically for respiratory patients. The model has been incorporated into a randomised, controlled clinical trial including 279 patients with COPD who had symptoms of anxiety. The patients were randomly allocated to either a group receiving $C B T$ with a respiratory nurse and selfhelp leaflets, or to a group receiving self-help leaflets alone. Analysis at 3-months followup showed that patients who received CBT alongside self-help leaflets had a significant improvement in anxiety symptoms compared to those who only received the leaflets. Furthermore, this delivery system was shown to be extremely cost effective and resulted in reduced attendance to the accident and emergency department and hospital admittance.

Addressing mental health and physical symptoms and encouraging physical activity are vital components required when developing self-management plans for COPD. Unlike respiratory nurses, many health professionals may not be able to address all key elements, prompting Dr Heslop-Marshall to state: "We need to take a leap from failure to success and start addressing the psychological needs of our patients. We either need to train our respiratory teams to do this or develop a digital treatment programme. Hopefully, we will be able to improve our patient's QoL and impact healthcare resources using this intervention."

\section{WALKING IN THE PATIENTS SHOES}

To reinforce the understanding that physical and psychological health are intertwined, Principal clinical psychologist Debra Sandford stressed the importance of increasing empathy and psychologically-focussed skills in patientcentred care. Clinically, empathy is the ability to step into the shoes of another person, aiming 
to understand their feelings and perspectives, and to use that understanding to guide our actions. Many studies have shown that increased empathy results

in better patient outcomes and greater patient satisfaction because empathy plays a strong role in creating a therapeutic alliance. Patient distress, which is generally under supported throughout a patient's treatment journey, is often connected to exacerbation. Stating that: "Empathy is about discovering what that person needs and giving that," Dr Sandford concluded by providing key traits of highly empathic people, including curiosity, challenging prejudice and preconceived ideas, active listening, inspiring others, and walking in someone else's shoes. Addressing this unmet need for empathy in clinical care may improve patient care and positively impact treatment adherence, mortality, and morbidity in patients, Dr Sandford emphasised.

\section{EARLIER PALLIATIVE CARE TO ADDRESS UNMET PHYSICAL AND PSYCHOLOGICAL NEEDS}

The final presentation by Prof Kathleen Lindell, Simmons Center for Interstitial Lung Disease, Pittsburgh, Pennsylvania, USA, discussed integrating early palliative care for patients with IPF and their caregivers. Patients with IPF often have unmet physical and psychological needs, and patient and caregiver perspectives have highlighted there is an increased need for support in this population. Even though patients generally have a good understanding of their disease prognosis, they are not fully aware of the management options that are available to them. This patient population has reported decreased QoL, overwhelming symptom burden, and frustrations with physical limitations, oxygen therapy, and caregiver burden.

Prof Lindell argued that palliative care is appropriate at any point in a serious illness and hypothesised that stress, symptom burden, poor QoL, and poor care planning for IPF patients and their caregiver can be overcome by early introduction of palliative care in the course of IPF.
In her study, she aimed to measure the feasibility of palliative care, as measured by recruitment and retention rate; determine the acceptability of the support intervention on knowledge regarding disease, self-management, and treatment strategies for patients and their caregivers; and assess the impact of their support intervention on stress, symptom burden, and QoL.

Intervention was given in the form of Symptom management, Understanding the disease, Pulmonary rehabilitation, Palliative care, Oxygen therapy, Research considerations, and Transplantation (SUPPORT) intervention to increase knowledge of the disease, teach self-management strategies, and facilitate preparedness with end of life planning. Results showed a recruitment rate of $56 \%$ and patients found the intervention to be acceptable. Data analysis assessing the impact of the SUPPORT intervention is still in progress, yet the plan to integrate palliative care into the care of patients with IPF and their caregivers is highly innovative and could potentially close the gap between patients with IPF and quality end of life care.

\section{CONCLUSION}

Given the unavailability of psychological support throughout a patient's journey, it is important to remember that 'one size doesn't fit all', and patients respond to different forms of support differently. However, increased focus on disease-specific fears and providing empathy, cognitive therapies, and palliative care, should be considered a priority because they have the potential to greatly reduce the psychological distress experienced by these patients. As Flewett stated in his patient perspective video, "communication is essential," and the best way to move forward is to clearly communicate with the patients and understand their unmet psychological needs. 